\title{
Ảnh hưởng của độ mở thương mại đến mức độ truyền dẫn tỷ giá hối đoái vào lạm phát ở Việt Nam
}

\section{The impact of trade openness on exchange rate pass through in Vietnam}

\author{
Quách Doanh Nghiệp ${ }^{1 *}$, Nguyễn Thị Ngọc Trang ${ }^{1}$, Nguyễn Hoàng Thụy Bích Trâm ${ }^{1}$ \\ ${ }^{1}$ Trường Đại học Kinh tế Thành phố Hồ Chí Minh, Việt Nam \\ "Tác giả liên hệ, Email: nghieptcdn@ueh.edu.vn
}

\section{THÔNG TIN}

DOI: 10.46223/HCMCOUJS. econ.vi.13.3.520.2018

Ngày nhận: 12/07/2018

Ngày nhận lại: 28/09/2018

Duyệt đăng: 02/10/2018

\section{Tù khóa:}

độ mở thương mại, hồi quy chuyển tiếp trơn (STR), truyền dẫn tỷ giá

Keywords:

exchange rate pass through, smooth transition regression model (STR), trade openness

\section{TÓM TẮT}

Nghiên cứu này sử dụng phương pháp Hồi quy chuyển tiếp trơn (STR) nhằm tìm kiếm bằng chứng về sự ảnh hưởng của độ mở thương mại lên mức độ truyền dẫn của tỷ giá hối đoái vào trong lạm phát. Kết quả cho thấy độ mở thương mại càng lớn thì càng làm giảm mức độ truyền dẫn của tỷ giá hối đoái vào trong lạm phát, điều này được giải thích bởi áp lực cạnh tranh đến từ các đối thủ nước ngoài hoặc có yếu tố nước ngoài trong quá trình mở cửa hội nhập ngày càng lớn của Việt Nam đã làm giảm sức mạnh định giá của các doanh nghiệp. Bài nghiên cứu tìm thấy ngưỡng độ mở thương mại mà tại đó quá trình truyền dẫn tỷ giá thay đổi là khoảng $117 \%$ GDP.

ABSTRACT
This paper investigates the impact of trade openness on the
pass-through of the exchange rate to inflation by using a smooth
transition regression model (STR). The result shows that the
greater the degree of trade openness is, the lower the exchange
rate pass-through is. This explains that the intensity of
competitiveness increases when foreign firms enter particular
industries in Vietnam and the rapid economic integration could
cause the reduction in local firms' pricing power. This result also
shows that changes in market competitiveness are the important
channels through which the exchange rate affects inflation. The
trade openness threshold, in which the exchange rate pass-
through coefficient changes, is 117\% of GDP.




\section{Giới thiệu}

Truyền dẫn tỷ giá (Exchange Rate Pass Through - ERPT) vào lạm phát luôn là một mối quan tâm lớn của những nhà làm chính sách, đặc biệt là Ngân hàng Trung ương. Bởi vì khi nắm bắt được cách thức và quy mô mà những biến động trong tỷ giá được chuyển vào trong lạm phát sẽ giúp Ngân hàng Trung ương thực thi chính sách bình ổn giá cả hiệu quả hơn thông qua khả năng dự báo được diễn tiến lạm phát trước các cú sốc trong tỷ giá.

Nogueira và León-Ledesma (2011) cho rằng sự thay đổi trong tỷ giá được chuyển vào trong các mức giá cả là điều cực kỳ quan trọng đối với những người làm chính sách. Những ảnh hưởng này không chỉ tác động lên mức lạm phát hiện tại mà còn tác động lên kỳ vọng lạm phát, việc thiết lập chính sách tiền tệ và khả năng điều chỉnh tỷ giá để cân bằng lại thâm hụt thương mại.

Theo Goldberg và Knetter (1996) truyền dẫn tỷ giá là phần trăm thay đổi trong giá nhập khẩu tính bằng đồng tiền nội tệ từ một phần trăm thay đổi trong tỷ giá giữa quốc gia xuất khẩu và quốc gia nhập khẩu. Theo thời gian, định nghĩa này đã được các nhà nghiên cứu kế thừa và mở rộng ra đối với giá sản xuất và giá tiêu dùng. Nghiên cứu này được thực hiện nhằm đánh giá sự ảnh hưởng của các yếu tố thuộc môi trường vĩ mô lên mức độ truyền dẫn của tỷ giá vào lạm phát. Các nghiên cứu hiện tại phần lớn tập trung đánh giá mối quan hệ truyền dẫn trực tiếp giữa tỷ giá và lạm phát mà chưa có nhiều nghiên cứu đánh giá về vai trò của các yếu tố thuộc môi trường vĩ mô - môi trường truyền dẫn - sẽ có những ảnh hưởng cụ thể như thế nào đến hệ số truyền dẫn. Theo chúng tôi cần phải đặt vấn đề truyền dẫn tỷ giá trong các điều kiện vĩ mô cụ thể theo đặc thù của từng quốc gia để đánh giá đầy đủ hơn quá trình và mức độ truyền dẫn. Với mục đích đó, nghiên cứu này xem xét ảnh hưởng của độ mở thương mại lên sự truyền dẫn của tỷ giá vào lạm phát ở Việt Nam. Chúng tôi xem xét đến điều này vì chúng thể hiện cho đặc điểm nền kinh tế nhỏ, mở của nền kinh tế Việt Nam trong quá trình hội nhập kinh tế toàn cầu. Quá trình toàn cầu hóa đang diễn ra mạnh mẽ nên sẽ có tác động đáng kể lên mức độ truyền dẫn tỷ giá, Việt Nam đang nằm trong dòng chảy nhộn nhịp của kinh tế thế giới, nền kinh tế có độ mở kinh tế ngày một cao, đó là nguyên nhân khiến chúng tôi muốn khảo sát yếu tố này sẽ ảnh hưởng như thế nào đến quá trình truyền dẫn tỷ giá vào lạm phát ở Việt Nam. 


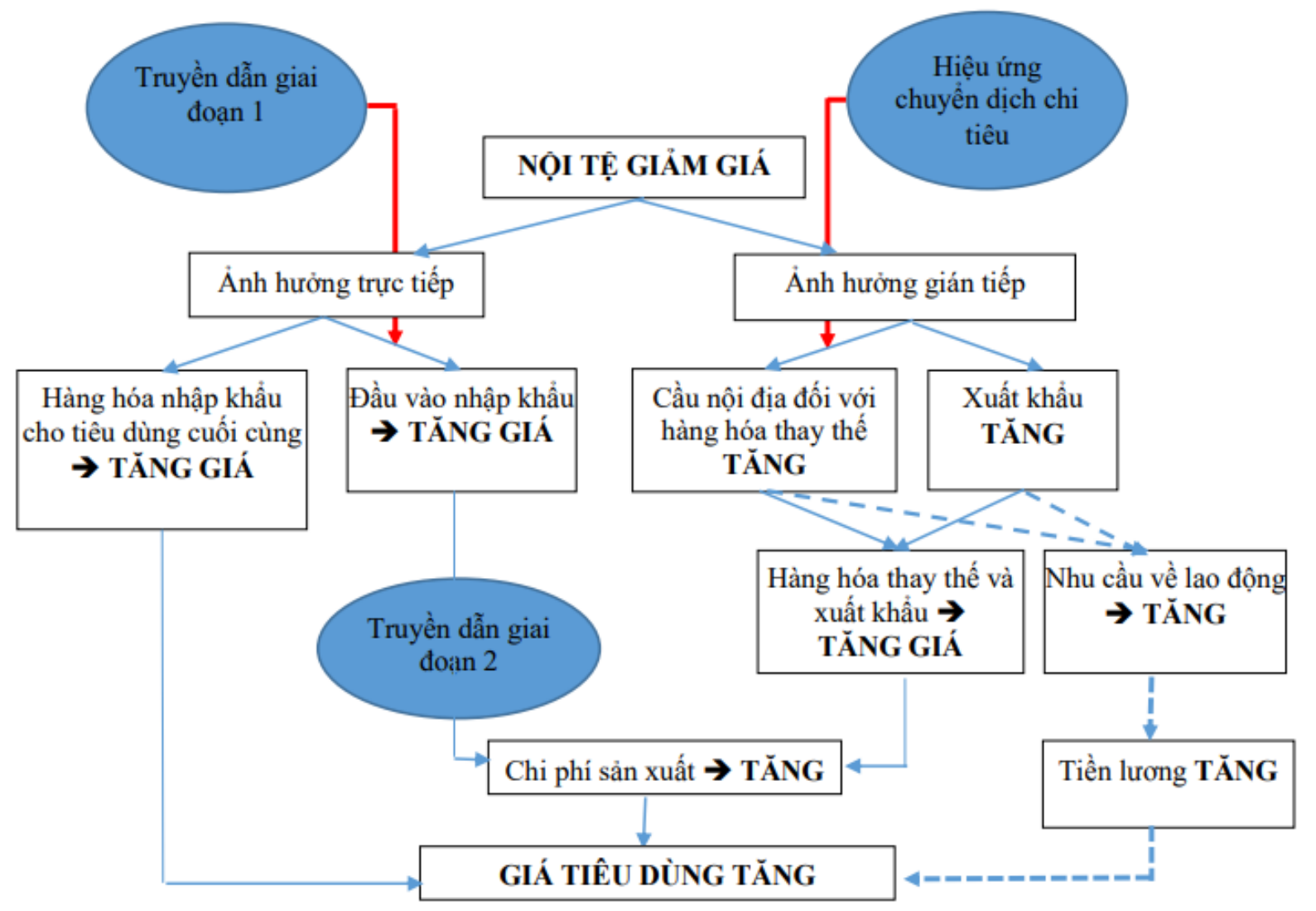

Hình 1. Sơ đồ truyền dẫn của tỷ giá vào giá tiêu dùng

Nguồn: Laflèche (1997) và tập hợp của tác giả.

Kết quả từ nghiên cứu sẽ là góp thêm thông tin quan trọng để cơ quan chức năng có thể dựa theo đó xây dựng các chính sách điều tiết thích hợp đến tỷ giá trong điều kiện nền kinh tế bị ảnh hưởng bởi hiện tượng đô la hóa một phần và mức độ mở cửa hội nhập ngày càng cao như ở Việt Nam. Chúng tôi hy vọng khi đặt mối quan hệ truyền dẫn tỷ giá vào lạm phát vào điều kiện môi trường vĩ mô cụ thể sát với điều kiện kinh tế của Việt Nam sẽ giúp bóc tách một phần mối quan hệ này ra khỏi hệ thống các mối quan hệ đan chéo phức tạp của nền kinh tế thực.

Nghiên cứu này được thực hiện nhằm trả lời cho câu hỏi nghiên cứu dữ liệu hệ số truyền dẫn của tỷ giá vào lạm phát có khác nhau ở những mức độ mở cửa thương mại khác nhau hay không?

Nghiên cứu được thực hiện ở Việt Nam, sử dụng dữ liệu hàng quý, thời gian nghiên cứu là 2002:Q2 - 2016:Q4. Nguồn dữ liệu được thu thập từ IMF, Data Stream của Thomson Reuters và Tổng cục thống kê Việt Nam.

\section{2. Ảnh hưởng của độ mở thương mại lên cơ chế truyền dẫn tỷ giá}

Khi một quốc gia tiến hành mở cửa thương mại sẽ gia tăng áp lực cạnh tranh giữa các doanh nghiệp khiến cho sức mạnh định giá của doanh nghiệp bị ảnh hưởng. Binici, Cheung, và Lai (2012) kết luận độ mở thương mại lớn hơn làm tăng sức mạnh cạnh tranh và làm giảm sức mạnh định giá của doanh nghiệp vì thế làm giảm lạm phát. 
Các doanh nghiệp nội địa trước áp lực cạnh tranh nâng cao được năng suất sản xuất, cải tiến công nghệ, tối ưu hóa các lợi thế cạnh tranh của mình từ đó tạo ra sản phẩm với mức chi phí thấp hơn, có thể góp phần làm giảm lạm phát. Thông thường khi đồng nội tệ bị mất giá sẽ khiến cho chi phí nhập khẩu tăng lên, kéo theo sự truyền dẫn những biến động tỷ giá vào trong giá cả của nền kinh tế tăng lên, nghĩa là truyền dẫn tỷ giá (Exchange rate pass through - ERPT) tăng khi đồng nội tệ mất giá. Tuy nhiên, dưới áp lực cạnh tranh từ mở cửa thương mại, các doanh nghiệp sẽ chần chừ trong việc chuyển những biến động trong tỷ giá vào giá bán, các doanh nghiệp có khuynh hướng chấp nhận lợi nhuận biên của họ giảm đi để giữ thị phần. Độ mở thương mại càng lớn, áp lực lên các doanh nghiệp càng lớn, vì vậy khi mức độ hội nhập thương mại càng cao có khuynh hướng kéo mức lạm phát giảm xuống do ERPT giảm. Điều này cho thấy độ mở thương mại gây ra hiệu ứng ngược chiều lên quá trình truyền dẫn tỷ giá vào lạm phát vì làm suy giảm sức mạnh định giá của doanh nghiệp. Nghiên cứu của Gust, Leduc, và Vigfusson (2010) tìm thấy trong điều kiện độ mở thương mại ngày càng tăng thì sự phản ứng của những nhà nhập khẩu đối với giá của các đối thủ cạnh tranh cũng tăng lên để giữ thị phần và qua đó làm giảm mức truyền dẫn. Điều này nghĩa là các nhà nhập khẩu (nội địa) luôn cân nhắc mỗi khi tăng giá do tỷ giá thay đổi bởi vì nếu họ làm điều đó mà đối thủ của họ không làm thì thị phần của họ có thể bị thu hẹp. Cho nên trong tình huống cạnh tranh ngày càng cao nhà nhập khẩu không vội vàng chuyển hết phần thay đổi trong tỷ giá vào trong giá bán mà chấp nhận gánh chịu để giữ giá bán như cũ qua đó ổn định thị phần, việc làm này sẽ làm biên lợi nhuận của họ giảm xuống.

Khi nền kinh tế mở rộng thương mại, nếu tỷ trọng hàng hóa nhập khẩu trong rổ hàng hóa tiêu dùng ít sẽ làm mức độ truyền dẫn thấp vì các công ty trong nước tránh điều chỉnh giá bán mỗi khi tỷ giá thay đổi vì chi phí thực đơn và vì vấn đề thị phần. Ngược lại, tỷ trọng hàng hóa nhập khẩu trong rổ hàng hóa tiêu dùng càng lớn (tức là tỷ lệ thâm nhập của hàng nhập khẩu cao hơn) thì mức độ truyền dẫn sẽ cao hơn. Bởi vì khi quốc gia quá phụ thuộc vào hàng hóa nước ngoài thì khi tỷ giá thay đổi, các nhà nhập khẩu sẽ tính phần thay đổi này vào giá bán và làm mức giá trong nền kinh tế tăng lên. Có thể thấy tỷ lệ nhập khẩu đầu vào trong sản xuất càng lớn, thì tác động của tỷ giá lên giá nhà sản xuất càng lớn. Do đó, độ mở thương mại của một nền kinh tế càng lớn thì hệ số truyền dẫn càng lớn theo Dornbusch, Fischer, và Samuelson (1977); Goldfajn và Werlang (2000); Adam và Cobham (2007). Mức độ hiện diện của các công ty nước ngoài trong nền kinh tế nội địa cao tỷ lệ thâm nhập của hàng hóa nhập khẩu đo lường bằng tỷ lệ hàng hóa nhập khẩu trong tiêu dùng gia tăng làm gia tăng mức độ truyền dẫn như kết quả của McCarthy (2007). Tương tự, An và Wang (2012) cũng cho rằng tỷ trọng nhập khẩu tăng đồng nghĩa với một sự thâm nhập sâu của hàng hóa nhập khẩu đồng thời phản ánh mức cạnh tranh kém của các doanh nghiệp nội địa. Trong tình huống này, các công ty nước ngoài hoặc các công ty nhập khẩu hàng hóa nước ngoài có thể chuyển những thay đổi trong tỷ giá vào nước nhập khẩu nhiều hơn từ đó làm gia tăng mức độ truyền dẫn.

Khundrakpam (2007) khi nghiên cứu về tác động của thay đổi kinh tế lên truyền dẫn tỷ giá đến giá nội địa tại Ấn Độ đã cho thấy các yếu tố tự do hóa kinh tế như là: sự gia tăng trong độ mở thương mại, cắt giảm hàng rào thuế quan, xóa bỏ các giới hạn về khối lượng, sự thay đổi trong thành phần nhập khẩu là nguyên nhân gây ra sự truyền dẫn bất cân xứng của tỷ giá đối 
với trường hợp tỷ giá tăng hoặc giảm hoặc những thay đổi lớn và nhỏ trong tỷ giá đến mức độ lạm phát.

Từ các nghiên cứu đã dẫn, có thể thấy mức độ truyền dẫn của tỷ giá vào trong giá cả tùy thuộc vào mức độ mở cửa thương mại của nền kinh tế. Cụ thể, khi độ mở thương mại ở mức thấp, biểu hiện bằng mức độ thâm nhập của hàng hóa nước ngoài vào trong nước thấp có thể sẽ dẫn đến mức độ truyền dẫn thấp, tuy nhiên khi độ mở thương mại cao thì truyền dẫn của tỷ giá sẽ cao, do hành vi của nhà sản xuất lúc này trở nên nhạy cảm với những biến động trong tỷ giá, tổng chi phí nhập khẩu đầu vào sẽ gia tăng tương xứng với tỷ lệ đầu vào nhập khẩu điều này sẽ khiến họ nhanh chóng điều chỉnh giá hàng hóa trong nền kinh tế.

\section{Phương pháp nghiên cứu, mô hình thực nghiệm và dữ liệu}

\subsection{Khung phân tích}

Chúng tôi xây dựng khung phân tích dựa theo Campa và Goldberg (2002), Nogueira và León-Ledesma (2011). Giá xuất khẩu được thiết lập bởi các công ty nước ngoài trong điều kiện thị trường cạnh tranh hoàn hảo và nhà xuất khẩu được giả định là luôn muốn tối đa hóa lợi nhuận của mình, khi đó giá hàng hóa tính theo đồng nội tệ sẽ được biểu diễn như sau:

$$
P_{i}^{h}=E \cdot C_{i}^{f}
$$

Trong đó $P_{i}^{h}$ là giá hàng hóa định danh bằng đồng tiền nội địa, $C_{i}^{f}$ là chi phí biên được tính bằng đồng ngoại tệ và $E$ là tỷ giá hối đoái được yết trực tiếp dưới dạng số lượng nội tệ tính trên một đơn vị ngoại tệ.

Theo lý thuyết mark-up, nhà xuất khẩu sẽ tính thêm trên giá xuất khẩu một đại lượng gọi là mark-up để tối đa hóa lợi nhuận của mình.

Gọi phần mark-up, được ký hiệu $\theta_{i}$, là đại lượng dựa theo chi phí biên $C_{i}^{f}$. Phần mark-

up được định nghĩa như sau: $\theta_{i} \equiv \frac{\eta_{i}}{\left(1-\eta_{i}\right)}$ trong đó $\eta_{i}$ là độ co giãn của cầu theo giá của hàng hóa i ở quốc gia nhập khẩu.

Bailliu và Fujii (2004) đã giả định rằng $\theta_{i}$ phụ thuộc vào áp lực cầu của thị trường tiêu thụ, do đó $\theta_{i}=\theta(Y)$ với Y là mức thu nhập (chi tiêu) ở quốc gia nhập khẩu.

Campa và Goldberg (2002) và Nogueira và León-Ledesma (2011) đã mở rộng giả định hàm mark-up khi cho rằng các doanh nghiệp sẽ phản ứng trước mức độ ổn định kinh tế vĩ mô của quốc gia nhập khẩu, chẳng hạn như khi lạm phát cao hoặc khi nền kinh tế đối mặt với khủng hoảng niềm tin thì ERPT sẽ cao hơn. Các tác giả cho rằng sự ổn định kinh tế vĩ mô của quốc gia nhập khẩu sẽ tác động lên quyết định truyền dẫn mức độ biến động của tỷ giá vào trong giá của doanh nghiệp dưới các kịch bản kinh tế vĩ mô khác nhau. Do đo, hàm mark-up mở rộng sẽ có dạng như sau:

$$
\theta_{i}=\theta\left(Y, E^{w(Z)}\right)
$$

Hàm mark-up theo quan điểm của Campa và Goldberg (2002), Nogueira và LeónLedesma (2011) được thể hiện dưới dạng phi tuyến thể hiện phản ứng của doanh nghiệp trước các yếu tố đại diện cho tính ổn định của môi trường vĩ mô. Trong nghiên cứu của Nogueira và 
León -Ledesma (2011) Z gồm hai trạng thái kinh tế tốt: lạm phát thấp và/hoặc mức độ tín nhiệm chính sách cao; trạng thái kinh tế xấu: lạm phát cao và/hoặc mức độ tín nhiệm chính sách thấp.

Hàm $\mathrm{W}(\mathrm{Z})$ được xem như số nhân mark-up theo cách sau: khi các công ty thiết lập giá trước cho một số thời kỳ thì phần mark-up sẽ phản ứng nhiều hơn nếu tỷ lệ lạm phát cao hoặc niềm tin của thị trường vào chính sách trở nên kém đi. Nói cách khác, trong môi trường lạm phát cao hoặc khủng hoảng niềm tin ERPT sẽ tăng lên.

Lấy logarit hai vế của phương trình (2) sẽ được cho dạng mô hình hồi quy ERPT như sau:

$$
p_{t}^{h}=\alpha+\beta e_{t}+w(Z) e_{t}+\psi y_{t}+\delta c_{t}^{*}+\varepsilon_{t}
$$

Từ phương trình trên, $\beta$ thể hiện mức độ truyền dẫn trực tiếp của tỷ giá vào mức giá và được kỳ vọng nằm giữa 0 và 1 . Nếu $\beta=1$, ERPT là hoàn toàn, nghĩa là nhà xuất khẩu sẽ chuyển dịch hoàn toàn những biến động của tỷ giá vào trong giá, chiến lược này thường được thể hiện dưới dạng giá hàng hóa tính bằng đồng ngoại tệ (foreign-currency pricing - FCP). Nếu $\beta=0$, nghĩa là ERPT bằng zero, xảy ra khi các công ty nước ngoài quyết định không thay đổi giá tính theo đồng tiền của thị trường tiêu thụ và hấp thụ toàn bộ biến động của tỷ giá vào trong phần mark-up, chiến lược này thường được gọi là thiết lập giá bằng đồng tiền địa phương (localcurrency pricing - LCP).

Có thể thấy, chiến lược định giá của các doanh nghiệp không chỉ phụ thuộc độc nhất vào điều kiện cầu nội địa. Chúng ta có thể nghĩ rằng doanh nghiệp nước ngoài có thể điều chỉnh giá sau biến động tỷ giá có liên quan đến nhiều yếu tố vĩ mô. Ví dụ, môi trường lạm phát như lập luận của Taylor (2000) có thể ảnh hưởng đến quy mô của ERPT. Một yếu tố quan trọng khác tác động lên kênh truyền ERPT đó là chu kỳ kinh doanh như trong nghiên cứu của Goldfajn và Werlang (2000).

Trong nghiên cứu này, chúng tôi giả sử rằng chiến lược định giá của doanh nghiệp nước ngoài tùy thuộc vào môi trường vĩ mô của nhà nhập khẩu trong một khung phân tích phi tuyến thể hiện qua hàm $\mathrm{k}(\mathrm{M})$. Khi thêm vào các yếu tố vĩ mô, chúng ta có thể viết lại hình dạng của phương trình mark-up của công ty nước ngoài như sau:

$$
\mu_{i}=\mu\left(Y, E^{K(M)}\right), \quad K(M) \geq 0
$$

Chúng ta có thể thu gọn phương trình (3) và (4) thông qua một phương trình hồi quy phi tuyến sau:

$$
p_{t}=\alpha+\beta e_{t}+\psi y_{t}+k(M) e_{t}+\delta w_{t}^{*}+\varepsilon_{t}
$$

Theo đó, hàm $\mathrm{k}(\mathrm{M})$ thể hiện kênh truyền dẫn gián tiếp phụ thuộc vào môi trường kinh tế vĩ mô. Chúng tôi giả sử rằng các nhân tố vĩ mô ảnh hưởng lên phần mark-up của các doanh nghiệp theo hướng phi tuyến. Do đó, chúng tôi giả định rằng tồn tại một số mức ngưỡng $\mathrm{M}$ phân chia nền kinh tế thành 2 trạng thái. Ví dụ, nếu yếu tố vĩ mô là mức độ lạm phát thì nền kinh tế được chia thành 2 trạng thái: môi trường lạm phát cao và môi trường lạm phát thấp.

$$
k(M)=\left\{\begin{array}{l}
0, \text { khi } M \leq M^{*} \\
\phi, \text { khi } M \geq M^{*}
\end{array}\right.
$$


Theo phương trình (5) và (6), mức độ truyền dẫn sẽ khác nhau và tùy thuộc vào biến số vĩ mô nằm trên hay dưới mức ngưỡng. Nếu quốc gia nhập khẩu có biến số vĩ mô có giá trị nhỏ (nhỏ hơn $M^{*}$ ) thì ERPT bằng $\beta$. Nếu biến số vĩ mô của quốc gia nhập khẩu đạt mức giá trị cao hơn mức ngưỡng thì hệ số ERPT sẽ là $\beta+\emptyset$. Chúng ta có thể thấy ERPT khác nhau tùy thuộc vào yếu tố đại diện cho trạng thái kinh tế vĩ mô nằm trên hay dưới mức ngưỡng. Thí dụ, trong các nghiên cứu đã cho thấy môi trường lạm phát cao hơn sẽ dẫn đến mức độ truyền dẫn cao hơn so với trạng thái lạm phát thấp. Vì vậy, điểm mạnh của phương trình (6) là có thể miêu tả được hành vi thay đổi trong tỷ giá theo cách thức phi tuyến, tương đối khác biệt so với cách tiếp cận của các nghiên cứu thực nghiệm trước đây. Cuối cùng, có một điều cũng cần được lưu ý là sự chuyển tiếp từ một trạng thái này sang một trạng thái khác được giả định là trơn (mượt).

\subsection{Phưong pháp nghiên cúu}

Chúng tôi dựa theo nghiên cứu của Shintani, Terada-Hagiwara, và Yabu (2013), Nogueira và León-Ledesma (2008) sử dụng lớp mô hình STR để ước lượng quy mô truyền dẫn, với giả thuyết nghiên cứu "Hệ số truyền dẫn của tỷ giá vào lạm phát sẽ thấp hơn trong giai đoạn nền kinh tế mở cửa nhiều hơn".

\subsubsection{Hàm hồi quy chuyển tiếp trơn}

Chúng tôi sử dụng khung phân tích hồi quy chuyển tiếp trơn (STR) được đưa ra bởi Van Dijk, Teräsvirta, và Franses (2002); Teräsvirta (2006) để thực hiện nghiên cứu.

Mô hình STR có thể được mô tả như hai mô hình tuyến tính bình quân trọng số, với trọng số được xác định bởi giá trị của hàm chuyển tiếp (Nogueira \& León-Ledesma, 2011). Mô hình STR tổng quát được xác định như sau.

$$
\begin{aligned}
y_{t} & =\phi^{\prime} z_{t}+\theta^{\prime} z_{t} \mathrm{G}\left(s_{t} ; \gamma, c\right)+u_{t} \\
& =[\phi+\mathrm{G}(\mathrm{s} t ; \gamma, c)]^{\prime} \mathrm{z} t+u t, \quad \text { với } t=1, \ldots, T
\end{aligned}
$$

Trong đó:

zt là véc tơ các biến giải thích, bao gồm véc tơ các trễ của biến nội sinh và véc tơ các biến ngoại sinh;

$\phi=\left(\phi_{0}, \phi_{1}, \ldots, \phi_{\mathrm{k}}\right)^{\prime}$ và $\theta=\left(\theta_{0}, \theta_{1}, \ldots, \theta_{\mathrm{k}}\right)^{\prime}$ là véc-tơ tham số với $(\mathrm{k}+1) \times 1$ tham số

$u_{t}$ là sai số tuân theo quy luật phân phối chuẩn;

$\mathrm{G}(\mathrm{s} t ; \gamma, c)$ là một hàm của biến chuyển tiếp $s t$ và bị chặn trong khoảng $(0,1)$; hàm số này liên tục mọi vị trí trong không gian tham số đối với bất kỳ giá trị $s t$;

Biến chuyển tiếp $s_{t}$ là một thành tố của $z_{t}$ do đó có thể được giả định là biến trễ nội sinh $\left(s_{t}=y_{t-d}\right)$ hoặc một biến ngoại sinh $\left(s_{t}=x_{k t}\right)$.

$\gamma$ là tham số độ dốc, chỉ tốc độ của hàm chuyển tiếp.

$c=\left(c_{1}, \ldots, c_{k}\right)^{\prime}$ là véc tơ các tham số ngưỡng, thỏa mãn $c_{1} \leq \cdots \leq c_{k}$, tham số ngưỡng này cho biết vị trí mà quá trình chuyển tiếp có thể xảy ra. Mô hình (1) có thể được hiểu như là một mô hình tuyến tính với hệ số thống kê biến đổi ngẫu nhiên theo thời gian: $\phi+(\mathrm{s} t ; \gamma, c)$ tùy thuộc vào giá trị của $s t$. 


\section{Có hai dạng hàm chuyển tiếp}

Nếu hàm chuyển tiếp là hàm logistic (LSTR) có dạng tổng quát

$G\left(s_{t} ; \gamma, c\right)=\left(1+\exp \left\{-\gamma \prod_{k=1}^{K}\left(s_{t}-c_{k}\right)\right\}\right)^{-1}, \gamma>0$

Mô hình LSTR (logistic......) được xác định như sau:

$$
\left\{\begin{array}{c}
y_{t}=\emptyset^{\prime} x_{t}+\theta^{\prime} x_{t} G\left(s_{t} ; \gamma, c\right)+u_{t} \\
G\left(s_{t} ; \gamma, c\right)=\left(1+\exp \left\{-\gamma \prod_{k=1}^{K}\left(s_{t}-c_{k}\right)\right\}\right)^{-1}
\end{array}\right.
$$

Các lựa chọn phổ biến trong thực nghiệm của $\mathrm{K}$ là $\mathrm{K}=1$ hoặc $\mathrm{K}=2$.

Các lựa chọn phổ biến là $\mathrm{K}=1$ và $\mathrm{K}=2$.

Đối với $\mathrm{K}=1$ : các tham số $\emptyset+\theta G\left(s_{t} ; \gamma, c\right)$ thay đổi đơn điệu như một hàm của $s_{t}$ từ $\emptyset$ đến $\emptyset+\theta$. Mô hình thu được gọi là LSTR1 sẽ tồn tại 1 ngưỡng, mô hình biểu thị cho trạng của hành vi bất đối xứng (asymmetry), tức là biến phụ thuộc thay đổi như thế nào khi biến chuyển tiếp ở dưới và ở trên giá trị ngưỡng.

Đối với $\mathrm{K}=2$ : hàm chuyển tiếp trở thành hàm bậc 2 , theo đó hàm chuyển tiếp $\mathrm{G}_{2}$ xứng quanh giá trị $(\mathrm{c} 1+\mathrm{c} 2) / 2$ và $\lim _{S_{t} \rightarrow \pm \infty} G_{2}=1 . \mathrm{G}_{2}$ không bao giờ bằng 0 ; giá trị tối thiểu của $\mathrm{G}_{2}$ nằm giữa 0 và 0,5 . Mô hình LSTR2 phù hợp để diễn tả một sự thay đổi nhỏ và lớn của biến chuyển tiếp $s_{t}$ quanh giá trị ngưỡng sẽ ảnh hưởng như thế nào đến biến phụ thuộc.

Nếu hàm chuyển tiếp là dạng hàm mũ (ESTR):

Hàm chuyển tiếp dạng mũ có phương trình tổng quát như sau:

$$
G\left(s_{t} ; \gamma, c\right)=1-\exp \left\{-\gamma\left(s_{t}-c_{1}^{*}\right)^{2}\right\}, \gamma>0
$$

Như giải thích của Christopoulos và León-Ledesma (2010) đối với cả hai mô hình, tham số $c$ được giải thích là ngưỡng giữa hai cơ chế tương ứng với $\mathrm{G}(\mathrm{s} t ; \gamma, c)=0$ và $\mathrm{G}(\mathrm{s} t ; \gamma, c)=1$. Đối với mô hình LSTR, các hệ số phi tuyến sẽ có giá trị khác nhau phụ thuộc vào việc biến chuyển tiếp thấp hơn hay cao hơn giá trị ngưỡng. Vì vậy, các hệ số $\phi^{\prime}+\theta^{\prime} \mathrm{G}(\mathrm{s} t ; \gamma, c)$ thay đổi một cách từ từ khi hàm số của $\mathrm{s}_{\mathrm{t}}$ từ $\phi^{\prime}$ đến $\phi^{\prime}+\theta^{\prime}$. Với ý nghĩa này, khi $\left(\mathrm{s}_{\mathrm{t}}-\mathrm{c}\right) \rightarrow-$ $\infty, \mathrm{G}(\mathrm{st} ; \gamma, \mathrm{c}) \rightarrow 0$ và các hệ số trở thành $\phi^{\prime}$, nếu $\left(\mathrm{s}_{\mathrm{t}}-\mathrm{c}\right) \rightarrow+\infty, \mathrm{G}(\mathrm{st} ; \gamma, \mathrm{c}) \rightarrow 1$ và các hệ số là $\left(\phi^{\prime}+\theta^{\prime}\right)$; và nếu st $=\mathrm{c}$, thì $\mathrm{G}(\mathrm{st} ; \gamma, \mathrm{c})=1 / 2$ và các hệ số sẽ là $\left(\phi^{\prime}+\theta^{\prime} / 2\right)$. Mô hình LSTR có sự chuyển tiếp diễn ra từ từ (mượt) giữa hai cơ chế. Một đặc điểm của mô hình LSTR là khi $\gamma \rightarrow \infty$, mô hình LSTR trở thành mô hình hồi quy ngưỡng, với sự chuyển tiếp diễn ra đột ngột. Nếu $\gamma \rightarrow 0$ thì hàm chuyển tiếp $G(s t ; \gamma, c) \equiv 0$ và mô hình LSTR trở thành mô hình hồi quy tuyến tính.

Trong mô hình ESTR những thay đổi của hệ số hồi quy phụ thuộc vào biến chuyển tiếp là xa hay gần giá trị ngưỡng, bất kể là chênh lệch $\left(\mathrm{s}_{\mathrm{t}}-\mathrm{c}\right)$ là dương hay âm. Do đó, hàm chuyển tiếp lũy thừa $\mathrm{G}(\mathrm{st} ; \gamma, \mathrm{c}) \rightarrow 1 \mathrm{khi}\left(\mathrm{s}_{\mathrm{t}}-\mathrm{c}\right) \rightarrow \pm \infty$ và các hệ số là $\phi^{\prime}+\theta^{\prime}$. Và nếu $\left(\mathrm{s}_{\mathrm{t}}=\mathrm{c}\right)$, $\mathrm{G}(\mathrm{st} ; \gamma, \mathrm{c}) \equiv 0$ và các hệ số trở thành $\phi^{\prime}$. Một hạn chế của kỹ thuật ESTR đó là khi $\gamma \rightarrow \infty$ và 
$\gamma \rightarrow 0$, mô hình hầu như trở thành tuyến tính và do đó nó không lồng một mô hình ngưỡng (với quá trình chuyển đổi đột ngột) như là một trường hợp đặc biệt.

\subsubsection{Quy trình xây dụng mô hình STR}

Quy trình xây dựng mô hình LSTR và ESTR gồm 3 bước như đề xuất của Franses và Van Dijk (2000):

Bước 1: Thiết lập mô hình hồi quy tuyến tính trên nền tảng lý thuyết và các nghiên cứu thực nghiệm liên quan.

Thực hiện kiểm định giả thuyết tuyến tính trên mô hình nền tảng ban đầu (giả thuyết H0: mô hình tuyến tính). Khi giả thuyết phi tuyến được chấp nhận thì tiến hành chọn biến chuyển tiếp $s_{t}$ phù hợp để hình thành hàm chuyển tiếp G.

Bước 2: Ước lượng các tham số trong mô hình với hàm chuyển tiếp đã có.

Bước 3: Đánh giá chất lượng mô hình.

\subsection{Mô hình thục nghiệm}

Dựa theo khung phân tích và mô hình STR tổng quát, chúng tôi sử dụng mô hình có dạng:

$$
\begin{gathered}
\pi_{t}=\alpha_{0}+\sum_{j=1}^{N} \lambda_{j} \cdot \pi_{t-j}+\sum_{j=0}^{N} \Psi_{j} \Delta p_{t-j}^{i m p}+\sum_{j=0}^{N} \delta_{j} \Delta y_{t-j} \\
+\sum_{j=0}^{N} \beta_{j} \cdot \Delta e_{t-j}+\left(\sum_{j=0}^{N} \Phi_{j} \Delta e_{t-j}\right) \cdot G\left(s_{t-i} ; \gamma, c\right)+\varepsilon_{t}
\end{gathered}
$$

Trong đó:

$\pi_{t}:$ lạm phát (CPI)

$\Delta p_{t}^{i m p}$ : thay đổi của giá nhập khẩu

$\Delta y_{t}$ : tốc độ tăng trưởng GDP

$\Delta e_{t}$ : sự thay đổi của tỷ giá

$\gamma$ : là tham số độ dốc chỉ tốc độ chuyển tiếp giữa hai miền tăng và giảm

$c$ : giá trị ngưỡng.

$s_{t}$ : biến chuyển tiếp

\section{Mô tả biến nghiên cứu}

Lạm phát được đo lường bằng phần trăm thay đổi trong chỉ số giá tiêu dùng CPI

IMP là giá nhập khẩu đại diện cho mức giá nhà xuất khẩu nước ngoài sẽ được tính bằng cách lấy bình quân gia quyền chỉ số lạm phát của các đối tác nước ngoài nhân với tỷ trọng thương mại song phương giữa Việt Nam và các nước này. 
Biến tỷ giá trong bài được sử dụng là mức tỷ giá danh nghĩa giữa VND và USD

$\Delta y_{t}$ : tốc độ tăng trưởng kinh tế được đại diện bởi tốc độ tăng trưởng GDP

Biến chuyển tiếp $\mathrm{s}_{\mathrm{t}}$ : là biến độ mở thương mại được đại diện bằng tổng xuất nhập khẩu/GDP.

\section{Kết quả nghiên cứu}

\section{1. Đôi nét về lạm phát và biến động tỷ giá hối đoái ở Việt Nam}

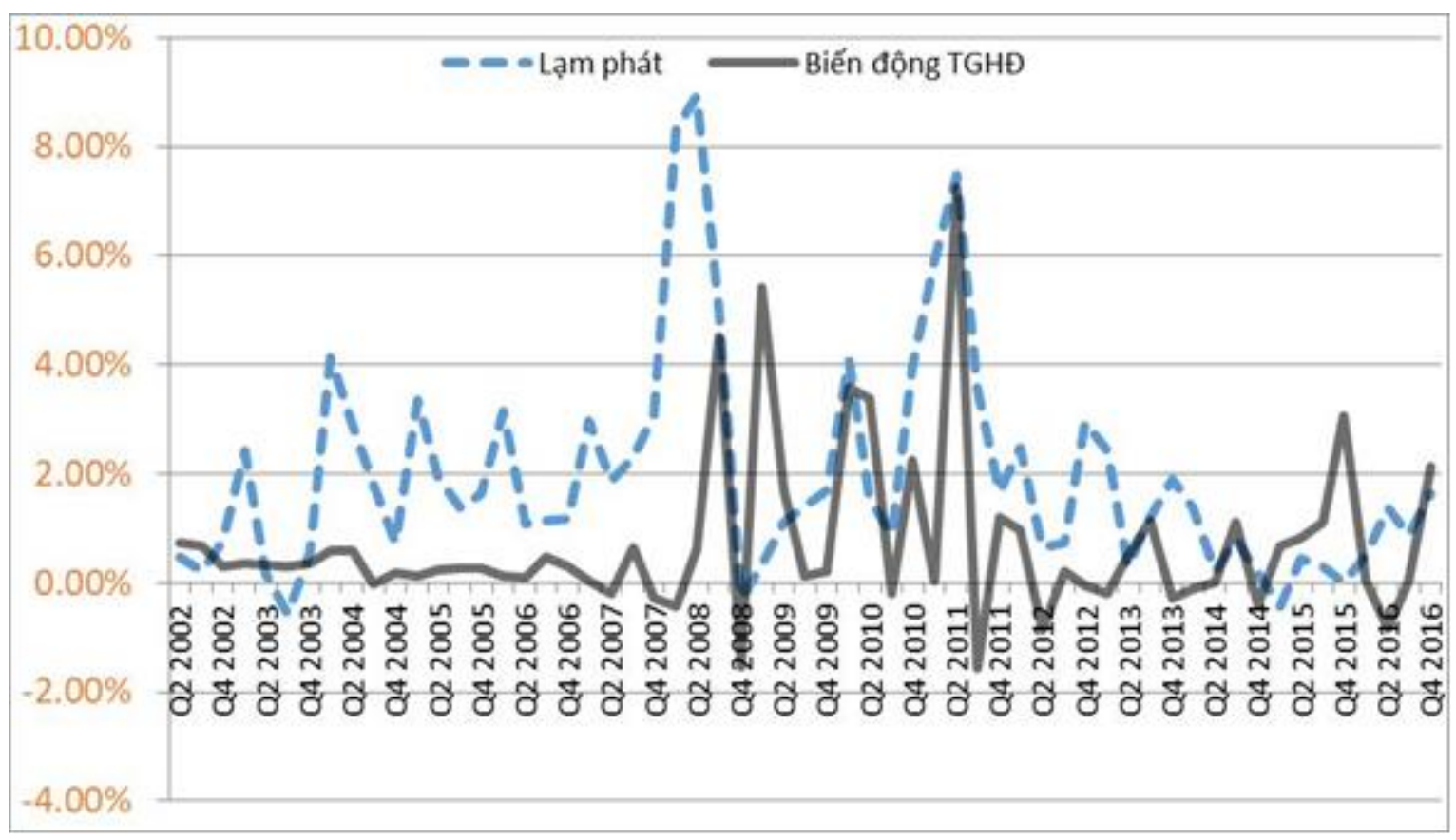

Hình 2. Mô tả biến lạm phát và biến động tỷ giá

Nguồn: IMF và Tổng cục thống kê

\subsubsection{Tình trạng lạm phát}

Tình trạng lạm phát của Việt Nam trong hơn gần 15 năm của giai đoạn nghiên cứu có nhiều biến động nhưng nhìn chung nằm trong khung khoảng $4 \%$ hàng quý. Trong khoảng thời gian từ 2002 đến 2016 thì thời điểm cuối năm 2007 đến nửa đầu năm 2008 lạm phát tăng vọt lên đến gần $9 \%$ tương ứng với giai đoạn nền kinh tế Mỹ nói riêng và toàn thế giới nói chung trong đó có Việt Nam bước vào thời kỳ kinh tế suy thoái và khủng hoảng. Giai đoạn thứ hai với đỉnh lạm phát tăng đến mức 7\% vào quý 2 năm 2011, năm được xem nhiều vấn đề vĩ mô xuất hiện nổi cộm và dày đặc. Trong bối cảnh đó, Chính phủ đã ban hành Nghị quyết số 11/2011/NQ$\mathrm{CP}$ về các nhóm giải pháp chủ yếu để thực hiện nhiệm vụ trọng tâm, cấp bách hiện nay là kiềm chế lạm phát, ổn định kinh tế vĩ mô, bảo đảm an sinh xã hội phần nào phản ánh tính cấp bách của tình hình và sự nhạy bén của chính phủ trong nhìn nhận và xử lý vấn đề kinh tế vĩ mô của Việt Nam. Tình hình lạm phát sau đó dần được kiểm soát và giai đoạn tiếp theo lạm phát đều nằm ở khung khoảng $3 \%$. 


\subsubsection{Mức độ biến động tỷ giá hối đoái}

Tỷ giá hối đoái USD/VND trong giai đoạn nghiên cứu nhìn chung có 3 trạng thái tương đối rõ rệt. Giai đoạn tư năm 2002 đến cuối năm 2007, tỷ giá "lặng sóng” mức độ biến động hầu như không đáng kể nếu không muốn nói là thời kỳ tỷ giá ổn định của Việt Nam. Tuy nhiên, giai đoạn từ đầu năm 2008 đến cuối năm 2011 là giai đoạn tỷ giá của Việt Nam biến động mạnh, 2 đỉnh tỷ giá được thiết lập vào năm 2008 và năm 2011 cũng gần như trùng nhịp với diễn tiến của lạm phát trong nền kinh tế lần lượt ở mức 5\% và 7\%. Giai đoạn sau năm 2011 biến động tỷ giá hàng quý xoay quanh khu vực $+/-3 \%$ tuy nhiên mức độ dao động nhiều hơn, phần nào phản ánh mức độ “nới lỏng” trong kiểm soát tỷ giá của Ngân hàng nhà nước.

\subsection{Thống kê mô tả và mối tương quan giữa biến nghiên cúu}

\section{Bảng 1}

Thống kê mô tả các biến

\begin{tabular}{|c|c|c|c|c|c|}
\hline Biến số & Số quan sát & $\begin{array}{c}\text { Giá trị Trung } \\
\text { bình }\end{array}$ & $\begin{array}{c}\text { Sai số } \\
\text { chuẩn }\end{array}$ & $\begin{array}{c}\text { Giá trị nhỏ } \\
\text { nhất }\end{array}$ & $\begin{array}{c}\text { Giá trị lớn } \\
\text { nhất }\end{array}$ \\
\hline inf_rate & 59 & 0.0195762 & 0.0199315 & -0.0055183 & 0.0896873 \\
\hline imp_rate & 59 & 0.0008115 & 0.0441214 & -0.1694431 & 0.1975357 \\
\hline GDP_rate & 59 & 0.3911562 & 0.7653943 & -0.7971994 & 1.462702 \\
\hline ex_rate & 59 & 0.0071258 & 0.0153651 & -0.0156605 & 0.0725734 \\
\hline openness & 59 & 0.8543966 & 0.5400396 & -0.0069041 & 2.197571 \\
\hline
\end{tabular}

Nguồn: Tác giả tự tính toán

\section{Bảng 2}

Ma trận hệ số tương quan giữa các biến

\begin{tabular}{|c|c|c|c|c|c|}
\hline & inf_rate & imp_rate & GDP_rate & ex_rate & openness \\
\hline inf_rate & 1.0000 & & & & \\
\hline imp_rate & 0.0615 & 1.0000 & & & \\
\hline GDP_rate & -0.1903 & -0.0085 & 1.0000 & & \\
\hline ex_rate & 0.1009 & $-0.2983^{* *}$ & -0.0944 & 1.0000 & \\
\hline openness & -0.0575 & -0.0028 & -0.1861 & 0.0236 & 1.0000 \\
\hline
\end{tabular}

Nguồn: Tác giả tự tính toán

Bởi vì hầu hết các hệ số tương quan đều thấp hơn 0.8 nên theo kinh nghiệm các biến trong nghiên cứu đều không cho thấy xảy ra khả năng đa cộng tuyến giữa các biến. 


\subsection{Kiểm định tính dùng, độ trễ thích hợp}

\subsubsection{Kiểm định tính dìng của biến nghiên cứu}

\section{Bảng 3}

Tính dừng của các biến

\begin{tabular}{|c|c|c|c|}
\hline & $\begin{array}{c}\text { Thống kê kiểm định (Test } \\
\text { Statistic) }\end{array}$ & p-value for Z(t) & Kết luận \\
\hline inf_rate & -3.801 & 0.0029 & Chuỗi dừng \\
\hline imp_rate & -10.731 & 0.0000 & Chuỗi dừng \\
\hline GDP_rate & -12.087 & 0.0000 & Chuỗi dừng \\
\hline exchange_rate & -4.793 & 0.0001 & Chuỗi dừng \\
\hline openness & -8.747 & 0.0000 & Chuỗi dừng \\
\hline
\end{tabular}

Các giá trị tới hạn (Critical value): 1\%: -3.569; 5\%: -2.924; 10\%: -2.597

Nguồn: Tác giả tự tính toán

Bảng 3 cho thấy kết quả kiểm định tính dừng các biến bằng kiểm định $\mathrm{ADF}$, tất cả các biến đều dừng.

\subsubsection{Kết quả ảnh hưởng của độ mở thwơng mại lên quá trình truyền dẫn tỷ giá}

Để thực hiện mô hình hồi quy chuyển tiếp trơn trước tiên chúng ta cần xác định độ trễ thích hợp cho mô hình hồi quy tuyến tính gốc. Việc chọn độ trễ cho biến độc lập và biến phụ thuộc đòi hỏi nhiều phép thử và so sánh kết quả từ mô hình để tìm được độ trễ phù hợp nhất. Trong bài nghiên cứu này chúng tôi sử dụng phần mềm Jmulti theo đề xuất của Terasvirta (2004) và hướng dẫn sử dụng phần mềm Jmulti của Kratzig (2005) để xây dựng mô hình STR. Theo đó, việc lựa chọn độ trễ của các biến được thực hiện từ cụ thể đến tổng quát, nghĩa là cho các cặp độ trễ kết hợp giữa biến độc lập và biến nghiên cứu để tìm kiếm mô hình phi tuyến phù hợp. Trong nghiên cứu này chúng tôi chọn độ trễ của biến độc lập và biến nghiên cứu lần lượt là 2 vì: thứ nhất, thỏa mãn tồn tại một mô hình phi tuyến để diễn tả mối quan hệ giữa các biến trong mô hình; thứ hai, mô hình LSTR1 được chọn thay vì LSTR2 bởi vì biến chuyển tiếp trong nghiên cứu này là độ mở thương mại (trade openness), mô hình chấp nhận một ngưỡng mà khi độ mở thương mại vượt quá sẽ chuyển mẫu nghiên cứu sang một trạng thái (regime) khác. Trong khi đó mô hình LSTR2 lại nghiên cứu một sự thay đổi xoay quanh giá trị ngưỡng của biến chuyển tiếp, điều này không phù hợp với đặc tính của biến trade openness nên chúng tôi không lựa chọn mô hình có độ trễ $(3,2)$. 


\section{Bảng 4}

Lựa chọn độ trễ cho mô hình

\begin{tabular}{|c|c|c|c|}
\hline Trễ của Y & Trễ của $\mathbf{X}$ & Biến chuyển tiếp & Mô hình \\
\hline 1 & 1 & openness(t-1)* & LSTR1 \\
\hline 2 & 1 & imp_rate_1(t-1)* & LSTR2 \\
\hline 3 & 1 & openness(t-1)* & LSTR1 \\
\hline 4 & 1 & openness(t-1)* & LSTR1 \\
\hline 5 & 1 & Không có & Linear \\
\hline 1 & 2 & openness $(t-1)^{*}$ & LSTR1 \\
\hline 2 & $\mathbf{2}$ & openness(t-1)* & LSTR1 \\
\hline 3 & 2 & openness(t-1)* & LSTR2 \\
\hline 4 & 2 & Không có & Linear \\
\hline 1 & 3 & Không có & Linear \\
\hline
\end{tabular}

Nguồn: Tác giả tự tính toán từ phần mềm Jmulti

\subsubsection{Kết quả mô hình hồi quy tuyến tính}

\section{Bảng 5}

Kết quả mô hình hồi quy tuyến tính

\begin{tabular}{|l|c|c|c|c|}
\hline & Hệ số & Sai số chuẩn & t-statistic & P_value \\
\hline Hằng số & 0.0113228 & 0.0044397 & 2.55 & 0.014 \\
\hline inf_rate $t-1$ & 0.9312303 & 0.1363181 & 6.83 & 0.000 \\
\hline inf_rate $t-2$ & -0.3263745 & 0.1418056 & -2.30 & 0.026 \\
\hline imp_rate $t$ & -0.1012772 & 0.0666846 & -1.52 & 0.136 \\
\hline imp_rate $t-1$ & 0.035393 & 0.0611673 & 0.58 & 0.566 \\
\hline imp_rate $t-2$ & 0.0595941 & 0.0481274 & 1.24 & 0.222 \\
\hline GDP_rate $t$ & -0.0107558 & 0.0031807 & -3.38 & 0.001 \\
\hline GDP_rate $t-1$ & -0.0017096 & 0.0040033 & -0.43 & 0.671 \\
\hline GDP_rate $t-2$ & 0.0012378 & 0.0031822 & 0.39 & 0.699 \\
\hline ex_ratet & 0.1215659 & 0.1285251 & 0.95 & 0.349 \\
\hline ex_rate $t-1$ & 0.2573789 & 0.1630155 & 1.58 & 0.121 \\
\hline ex_ratet-2 & -0.2044215 & 0.1799574 & -1.14 & 0.262 \\
\hline
\end{tabular}




\begin{tabular}{|l|c|l|l|l|}
\hline & Hệ số & Sai số chuẩn & t-statistic & P_value \\
\hline F(11, 45) & 6.60 & & & \\
\hline Prob > F & 0.0000 & & & \\
\hline Adj R-squared & 0.6174 & & & \\
\hline
\end{tabular}

Nguồn: Tác giả tự tính toán từ phần mềm Stata

Hệ số truyền dẫn ERPT từ mô hình hồi quy tuyến tính trong ngắn hạn là $0,12 \%$. Nghĩa là khi tỷ giá biến động $1 \%$ thì lạm phát biến động khoảng $0,12 \%$.

\subsubsection{Kết quả hồi quy phi tuyến}

\section{Bảng 6}

Kết quả hồi quy phi tuyến với biến chuyển tiếp $(\mathrm{s} t$ ) là biến độ mở thương mại (openness $\mathrm{t}-1$ )

\begin{tabular}{|l|c|c|c|c|}
\hline & Hệ số & Sai số chuẩn & t-statistic & P_value \\
\hline Phần tuyến tính & & & & \\
\hline Gamma $(\gamma)$ & 7.89371 & 5.1799 & 1.5239 & 0.1356 \\
\hline Ngưỡng (c) & 1.17479 & 0.0730 & 16.0846 & 0.0000 \\
\hline Constant & 0.06381 & 0.0376 & 1.6950 & 0.0980 \\
\hline inf_rate t-1 & 0.95562 & 0.1577 & 6.0592 & 0.0000 \\
\hline inf_rate t-2 & -0.31078 & 0.1585 & -1.9610 & 0.0571 \\
\hline ex_rate t & 0.30954 & 0.2205 & 1.4041 & 0.1682 \\
\hline ex_rate t-1 & 0.40284 & 0.2147 & 1.8759 & 0.0682 \\
\hline ex_rate t-2 & -0.20059 & 0.2099 & -0.9556 & 0.3451 \\
\hline imp_rate t & -0.10967 & 0.0716 & -1.5325 & 0.1335 \\
\hline imp_rate t-1 & 0.05024 & 0.0675 & 0.7439 & 0.4614 \\
\hline imp_rate t-2 & 0.06825 & 0.0529 & 1.2897 & 0.2047 \\
\hline GDP_rate t & -0.06314 & 0.0382 & -1.6537 & 0.1062 \\
\hline GDP_rate t-1 & -0.02788 & 0.0179 & -1.5578 & 0.1274 \\
\hline GDP_rate t-2 & -0.01056 & 0.0086 & -1.2335 & 0.2248 \\
\hline Phần phi tuyến & -0.07580 & 0.0542 & -1.3988 & 0.1698 \\
\hline Constant & -0.24944 & 0.3271 & -0.7626 & 0.4503 \\
\hline ex_rate t & -0.10436 & 0.3803 & -0.2744 & 0.7852 \\
\hline ex_rate t-1 & & & & \\
\hline
\end{tabular}




\begin{tabular}{|l|c|c|c|c|}
\hline & Hệ số & Sai số chuẩn & t-statistic & P_value \\
\hline ex_rate t-2 & -0.18017 & 0.4698 & -0.3835 & 0.7035 \\
\hline adjusted $R 2$ & 0.6620 & & & \\
\hline AIC & $-8.2641 \mathrm{e}+00$ & & & \\
\hline Nonlinear short-run ERPT & & & & \\
$G($ transition function $)=0$ & 0.3095 & & \\
$G($ transition function $)=1$ & 0.0601 & & \\
\hline Nonlinear Long-run ERPT & & & & \\
$G$ (transition function $)=0$ & 1.4410 & & & \\
$G($ transition function $)=1$ & -0.0625 & & & \\
\hline
\end{tabular}

Nguồn: Tác giả tự tính toán từ phần mềm Jmulti

Đối với mô hình LSTR,

Nếu $\left(s_{t}-c\right) \rightarrow-\infty$, tức là khi đó hàm $G \rightarrow 0$ hệ số truyền dẫn lần luợt là:

Hệ số truyền dẫn trong ngắn hạn:

$$
S R E R P T=\beta_{0}
$$

Hệ số truyền dẫn trong dài hạn:

$$
L R E R P T=\frac{\sum_{j=0}^{N} \beta_{j}}{1-\sum_{j=1}^{N} \lambda_{j}}
$$

Nếu $\left(s_{t}-c\right) \rightarrow+\infty$, tức là khi đó hàm $G \rightarrow 1$ hệ số truyền dẫn lần luợt là:

Hệ số truyền dẫn trong ngắn hạn:

$$
S R E R P T=\beta_{0}+\emptyset_{0} G\left(s_{t} ; \gamma, c\right)
$$

Hệ số truyền dẫn trong dài hạn:

$$
L R E R P T=\frac{\sum_{j=0}^{N} \beta_{j}+\sum_{j=0}^{N} \emptyset_{j} G\left(s_{t} ; \gamma, c\right)}{1-\sum_{j=1}^{N} \lambda_{j}}
$$

Kết quả từ phương trình hồi quy phi tuyến cho thấy, hệ số ERPT ngắn hạn phần tuyến tính là $0,3095 \%$ cao hơn mức truyền dẫn thu được từ mô hình hồi quy tuyến tính gốc.

Bảng 6 cho thấy tồn tại một mức ngưỡng của độ mở thương mại là 117,48\%GDP, điều này cho thấy hệ số truyền dẫn ERPT sẽ thay đổi khi biến chuyển tiếp vượt qua mức ngưỡng này.

Khi tính vào phần phi tuyến sau khi biến chuyển tiếp độ mở thương mại vượt qua giá trị ngưỡng $117,48 \%$ GDP thì ta có hệ số truyền dẫn ERPT tổng hợp như sau: $0,3095 \%$ + ($0,2494 \%)=0,0601 \%$. Điều này cho thấy khi độ mở thương mại vượt ngưỡng $117,48 \%$ GDP 
thì hệ số truyền dẫn ERPT sẽ giảm xuống, nghĩa là mức độ dịch chuyển những thay đổi của tỷ giá vào trong giá sẽ giảm khi độ mở thương mại gia tăng. Kết quả này tương đồng với các kết quả được tìm thấy bởi Binici và cộng sự (2012) hay Gust và cộng sự (2010). Kết quả này cung cấp thêm bằng chứng ở Việt Nam, khi độ mở thương mại lớn hơn có thể làm tăng súc mạnh cạnh tranh trong thị trường nội địa và làm giảm súc mạnh định giá của doanh nghiệp vì thế có thể làm giảm mức độ truyền dẫn cú sốc của tỷ giá vào trong giá từ đó dẫn đến giảm lạm phát. Nghĩa là trước sức ép từ quá trình mở cửa thương mại, ngày càng xuất hiện nhiều các đối thủ cạnh tranh có nguồn gốc từ nước ngoài đã buộc các doanh nghiệp nội địa giảm phần tăng thêm vào giá bán (mark-up) để giữ thị phần của mình, từ đó làm giảm sự trung chuyển những thay đổi khi đồng nội tệ giảm giá vào trong giá cả. Khi nền kinh tế có độ mở ngày càng lớn thì khi đồng nội tệ mất giá sức ép lên các doanh nghiệp nội địa càng lớn và khả năng chịu tổn thương cũng trở nên lớn hơn.

\subsubsection{Các điểm định chấp nhận mô hình}

\section{Bảng 7}

Kiểm định không có tự tương quan phần dư

\begin{tabular}{|c|c|c|c|c|}
\hline Độ trễ & Thống kê F & df1 & df2 & p-value \\
\hline 1 & 0.0421 & 1 & 37 & 0.8385 \\
\hline 2 & 0.2386 & 2 & 35 & 0.7890 \\
\hline 3 & 0.1458 & 3 & 33 & 0.9317 \\
\hline 4 & 0.4262 & 4 & 31 & 0.7885 \\
\hline
\end{tabular}

Nguồn: Kết quả phân tích dữ liệu của nhóm nghiên cứu

Các giá trị $\mathrm{p}$-value thu được từ thống kê $\mathrm{F}$ của kiểm định Godfrey với các độ trễ từ 1 đến 4 đều dẫn đến chấp nhận giả thuyết rằng không tồn tại tự tương quan trong phần sai số của mô hình.

\section{Bảng 8}

Kiểm định không còn phần phi tuyến

\begin{tabular}{|l|c|c|c|c|}
\hline Biến chuyển tiếp & \multicolumn{1}{|c|}{ F } & F4 & F3 & F2 \\
\hline inf_rate_2(t-1) & $1.5978 \mathrm{e}-01$ & $5.0443 \mathrm{e}-01$ & $2.4746 \mathrm{e}-01$ & $3.8345 \mathrm{e}-03$ \\
\hline inf_rate_2(t-2) & $1.8099 \mathrm{e}-02$ & $1.9082 \mathrm{e}-01$ & $9.9661 \mathrm{e}-03$ & $1.6392 \mathrm{e}-02$ \\
\hline imp_rate_1(t) & $3.9795 \mathrm{e}-03$ & $1.8016 \mathrm{e}-02$ & $2.2639 \mathrm{e}-02$ & $1.9057 \mathrm{e}-01$ \\
\hline ex_rate_IMF(t) & $4.6659 \mathrm{e}-02$ & $1.8195 \mathrm{e}-02$ & $8.7416 \mathrm{e}-01$ & $4.9654 \mathrm{e}-01$ \\
\hline openness(t) & $3.3129 \mathrm{e}-02$ & $3.8767 \mathrm{e}-02$ & $2.3443 \mathrm{e}-01$ & $2.8601 \mathrm{e}-01$ \\
\hline GDP_rate_1(t) & $1.1590 \mathrm{e}-02$ & $2.0452 \mathrm{e}-01$ & $1.5269 \mathrm{e}-03$ & $3.1965 \mathrm{e}-02$ \\
\hline imp_rate_1(t-1) & $4.3125 \mathrm{e}-01$ & $6.9865 \mathrm{e}-01$ & $7.4090 \mathrm{e}-02$ & $4.6339 \mathrm{e}-01$ \\
\hline ex_rate_IMF(t-1) & $6.2686 \mathrm{e}-01$ & $4.8125 \mathrm{e}-01$ & $7.9363 \mathrm{e}-01$ & $4.3483 \mathrm{e}-01$ \\
\hline openness(t-1) & $1.6782 \mathrm{e}-03$ & $7.3380 \mathrm{e}-03$ & $4.0026 \mathrm{e}-01$ & $1.0443 \mathrm{e}-04$ \\
\hline GDP_rate_1(t-1) & $6.0062 \mathrm{e}-02$ & $9.3367 \mathrm{e}-02$ & $7.0165 \mathrm{e}-01$ & $1.2685 \mathrm{e}-02$ \\
\hline imp_rate_1(t-2) & $3.1150 \mathrm{e}-01$ & $3.1886 \mathrm{e}-01$ & $2.6714 \mathrm{e}-01$ & $6.1391 \mathrm{e}-01$ \\
\hline ex_rate_IMF(t-2) & $1.1018 \mathrm{e}-01$ & $1.2159 \mathrm{e}-01$ & $2.1858 \mathrm{e}-01$ & $5.4124 \mathrm{e}-01$ \\
\hline
\end{tabular}




\begin{tabular}{|l|c|c|c|c|}
\hline Biến chuyển tiếp & F & F4 & F3 & F2 \\
\hline openness(t-2) & $6.6528 \mathrm{e}-01$ & $8.5133 \mathrm{e}-01$ & $1.7256 \mathrm{e}-01$ & $1.9655 \mathrm{e}-01$ \\
\hline GDP_rate_1(t-2) & $2.7184 \mathrm{e}-02$ & $1.9007 \mathrm{e}-01$ & $4.1758 \mathrm{e}-03$ & $2.2061 \mathrm{e}-01$ \\
\hline
\end{tabular}

Nguồn: Kết quả phân tích dữ liệu của nhóm nghiên cứu

Kiểm định $\mathrm{F}$ được sử dụng cho mô hình hồi quy phụ có bổ sung một hàm chuyển tiếp giả định khác vào trong mô hình hồi quy gốc ban đầu:

$$
y_{t}=\phi^{\prime} z_{t}+\theta^{\prime} z_{t} G\left(s_{1 t} ; \gamma_{1}, c_{1}\right)+\partial^{\prime} z_{t} H\left(s_{2 t} ; \gamma_{2}, c_{2}\right)+u_{t}
$$

Với $H\left(s_{2 t} ; \gamma_{2} ; c_{2}\right)$ là một hàm chuyển tiếp khác. Để kiểm tra xem phần dư có còn chứa thành phần phi tuyến nào bị bỏ sót hay không thì hàm $\mathrm{H}$ sẽ được triển khai theo xấp xỉ Taylor bậc ba dưới dạng:

$$
y_{t}=\phi^{\prime z_{t}}+\theta^{\prime z} \mathrm{r} G\left(s_{1 t} ; \gamma_{1}, c_{1}\right)+\alpha_{1}^{\prime} z_{t} s_{2 t}+\alpha_{2}^{\prime} z_{t} s_{2 t}^{2}+\alpha_{3}^{\prime} z_{t} s_{3 t}^{3}+\varepsilon_{t}^{\prime}
$$

Giả thiết cần kiểm định là $H_{n}: \propto_{1}=\propto_{2}=\propto_{2}=0$, kiểm định $\mathrm{F}$ được sử dụng như trường hợp tuyến tính bình thường. Kết quả từ Bảng 8 cho thấy các giá trị tính toán của $\mathrm{F}$ so với giá trị tra bảng đều chấp nhận giả thiết $\mathrm{H} 0$, điều này đồng nghĩa với việc không tồn tại hàm chuyển tiếp nào khác cho nên phần dư không còn phần phi tuyến bị bỏ sót.

\section{Thảo luận kết quả nghiên cứu và hàm ý chính sách}

Nghiên cứu của chúng tôi cung cấp thêm bằng chứng các yếu tố thuộc môi trường vĩ mô có tác động nhất định đến mức độ truyền dẫn của tỷ giá vào lạm phát. Theo đó độ mở cửa thương mại càng cao thì càng làm cho hệ số truyền dẫn giảm. Kết quả nghiên cứu tìm thấy cũng tương đồng với các lý thuyết và một số bằng chứng thực nghiệm đã được thực hiện trên thế giới.

Mở cửa thương mại, hội nhập với thế giới đã và đang trở thành một xu hướng không thể chối bỏ, trong bối cảnh đó mức độ cạnh tranh giữa doanh nghiệp trong nước và các doanh nghiệp có yếu tố nước ngoài sẽ ngày càng quyết liệt hơn nữa, sức mạnh định giá của các doanh nghiệp này có thể sẽ giảm xuống mỗi khi đồng nội tệ bị mất giá so với ngoại tệ vì lý do cần giảm phần mark-up để giữ thị phần và duy trì năng lực cạnh tranh. Ở góc độ điều hành của chính phủ, cần thiết phải giữ ổn định tỷ giá và không để đồng nội tệ mất giá quá nhiều sẽ giúp các doanh nghiệp nội địa có thêm tiềm lực để đương đầu với sức ép cạnh tranh từ các doanh nghiệp có nguồn gốc hoặc liên quan đến yếu tố nước ngoài.

\section{Tài liệu tham khảo}

Adam, C., \& Cobham, D. (2007). Exchange rate regimes and trade. The Manchester School, 75, 44-63.

An, L., \& Wang, J. (2012). Exchange rate pass-through: Evidence based on vector autoregression with sign restrictions. Open Economies Review, 23(2), 359-380.

Bailliu, J., \& Fujii, E. (2004). Exchange rate pass-through and the inflation environment in industrialized countries: An empirical investigation. Retrieved May 12, 2018, from https://ideas.repec.org/p/bca/bocawp/04-21.html 
Binici, M., Cheung, Y. W., \& Lai, K. S. (2012). Trade openness, market competition, and inflation: Some sectoral evidence from OECD countries. International Journal of Finance \& Economics, 17(4), 321-336.

Campa, J. M., \& Goldberg, L. S. (2002). Exchange rate pass-through into import prices: A macro or micro phenomenon? (NBER Working Papers, No 8934). Retrieved May 13, 2018, from https://econpapers.repec.org/paper/nbrnberwo/8934.htm

Christopoulos, D., \& León-Ledesma, M. A. (2010). Current account sustainability in the US: What did we really know about it? Journal of International Money and Finance, 29(3), 442-459.

Dornbusch, R., Fischer, S., \& Samuelson, P. A. (1977). Comparative advantage, trade, and payments in a Ricardian model with a continuum of goods. The American Economic Review, 67(5), 823-839.

Franses, P. H., \& Van Dijk, D. (2000). Non-linear time series models in empirical finance. Cambridge, UK: Cambridge University Press.

Goldberg, P. K., \& Knetter, M. M. (1996). Goods prices and exchange rates: What have we learned? Retrieved May 15, 2018, from https://econpapers.repec.org/paper/nbrnberwo/5862.htm

Goldfajn, I., \& Werlang, S. (2000). The pass-through from depreciation to inflation: A panel study. Retrieved May 15, 2018, from https://www.econbiz.de/Record/the-pass-throughfrom-depreciation-to-inflation-a-panel-study-goldfajn-ilan/10001741757

Gust, C., Leduc, S., \& Vigfusson, R. (2010). Trade integration, competition, and the decline in exchange-rate pass-through. Journal of Monetary Economics, 57(3), 309-324.

Khundrakpam, J. (2007). Economic reforms and exchange rate pass-through to domestic prices in India. Retrieved May 17, 2018, from https://www.bis.org/publ/work225.htm

Kratzig, M. (2005). STR analysis in JMulTi. Retrieved October 10, 2017, from http://www.jmulti.de/download/help/str.pdf

Laflèche, T. (1997). The impact of exchange rate movements on consumer prices. Bank of Canada Review, 1996(Winter), 21-32.

McCarthy, J. (2007). Pass-through of exchange rates and import prices to domestic inflation in some industrialized economies. Eastern Economic Journal, 33(4), 511-537.

Nogueira, R. P., Jr., \& León-Ledesma, M. A. (2008). Exchange rate pass-through into inflation: The role of asymmetries and non-linearities: Department of Economics Discussion Paper, University of Kent. Retrieved May 17, 2018, from https://www.econstor.eu/bitstream/10419/68099/1/559311389.pdf

Nogueira, R. P., Jr., \& León-Ledesma, M. A. (2011). Does exchange rate pass-through respond to measures of macroeconomic instability? Journal of Applied Economics, 14(1), 167180. 
Shintani, M., Terada-Hagiwara, A., \& Yabu, T. (2013). Exchange rate pass-through and inflation: A nonlinear time series analysis. Journal of international Money and Finance, $32,512-527$.

Taylor, J. B. (2000). Low inflation, pass-through, and the pricing power of firms. European Economic Review, 44(7), 1389-1408.

Terasvirta, T. (2004). Smooth transition regression modeling. In H. Lütkepohl \& M. Kratzig (Eds.), Applied time series econometrics (pp. 222-242). Cambridge, UK: Cambridge University Press.

Teräsvirta, T. (2006). Forecasting economic variables with nonlinear models. Handbook of Economic Forecasting, 1, 413-457.

Tran, T. N., \& Nguyen, T. N. T. (2015). Truyền dẫn tỉ giá hối đoái ở Việt Nam dưới tác động của môi trường lạm phát [Exchange rate transmission in Vietnam under the impact of inflationary environment]. Tạp chí Phát triển Kinh tế, 26(10), 51-71.

Van Dijk, D., Teräsvirta, T., \& Franses, P. H. (2002). Smooth transition autoregressive models -A survey of recent developments. Econometric Reviews, 21(1), 1-47. 